\title{
Praxiología Motriz y Fútbol: Lógica Interna e indicativos al proceso de enseñanza- aprendizaje-entrenamiento
}

\author{
Cesar Vieira Marques Filho \\ Universidade Estadual de Campinas, Brasil \\ cesarvmf@hotmail.com \\ Paulo Cesar Montagner \\ Universidade Estadual de Campinas, Brasil \\ Joäo Francisco Magno Ribas \\ Universidade Federal de Santa Maria, Brasil
}

Motor Praxeology and Soccer: Internal Logic and indications to the teaching-learning-training process

\begin{abstract}
Resumen:
El trabajo pedagógico con el Fútbol está insertado en contextos distintos y puede ser conducido de variadas maneras y, frecuentemente, está basado por el conocimiento empírico de entrenador en cuanto practicante de la modalidad. En esta investigación, los conocimientos de la Praxiología Motriz son utilizados para proporcionar un amplio entendimiento de la Lógica Interna del Fútbol. Por medio de un análisis praxiológico, construimos una base de conocimiento científico acerca del juego y proponemos indicativos al proceso de enseñanza-aprendizaje-entrenamiento de la modalidad. Los aspectos comunicacionales del juego, la dinámica de los cambios de roles y la caracterización de las Acciones Motrices ganan destaque acerca de una mayor base sobre las condiciones de juego. De esta manera, buscamos fomentar el conocimiento acerca del Fútbol y aportar para una selección coherente de métodos y contenidos por parte de los entrenadores.
\end{abstract}

Palabras ClaVe: Fútbol, Praxiología Motriz, Enseñanza-Aprendizaje-Entrenamiento.

\section{Abstract:}

The Soccer pedagogical work is inserted in different contexts and can be conducted in various ways. It is often supported by the empirical knowledge of coaches as practitioners of the sport. In this research, the knowledge of Motor Praxeology is used to provide a wider understanding of Soccer's Internal Logic. Through a praxiological analysis, we construct a scientific knowledge base about the game and propose indications to the teaching-learning-training process of this modality. The communicative aspects of the game, the dynamics of the role changes and the characterization of the motory actions are highlighted on a more intimate basis on the playing conditions. In this way, we seek to foster knowledge about Soccer and contribute to a coherent selection of methods and content by the coaches.

KEYWORDS: Soccer, Motor Praxeology, Teaching-Learning-Training.

\section{INTRODUCCIÓN}

La Praxiología Motriz consiste en una teoría cuya finalidad es profundizar el conocimiento sobre los juegos y deportes. Fue creada en la década de 1960, por el profesor francés Pierre Louis Parlebas, cuya formación contempla la Educación Física, la Lingüística, la Psicología y la Sociología (Saravi, 2012). En la construcción de la Praxiología Motriz, utilizó la relación de los conocimientos de la psicología social, la lingüística y las matemáticas aplicadas, direccionadas a las ciencias sociales, pues comprende el juego como una manifestación social. En 1999, el autor publicó la principal obra de la Praxiología Motriz: "Jeux, Sports et Sociétés: Lexique de Praxéolgie Motrice” dispuesta en forma de léxico. En 2001, hubo el lanzamiento de la versión en español, accionando como catalizador a la popularización de la teoría. La Praxiología Motriz se 
presenta como la "Ciencia de la Acción Motriz y especialmente de las condiciones, modos de funcionamiento y resultados de su desarrollo" (Parlebas, 2001, 354).

Una serie de estudios asocia la Praxiología Motriz y el Fútbol (Hernández Moreno, 2001; Mancila y Pozo, 2013; Marques Filho, Schmitz Filho, Ribas \& Bettega, 2017; Puig, 2011; Torres, 2013). Mientras tanto, en todos los casos, la Praxiología Motriz es empleada como instrumento de análisis para momentos específicos del juego, como pelotas paradas, pases, movimientos de algunos jugadores, participaciones del arquero e incidencia reglamentares. Una literatura que busque concebir el juego de manera más amplia, analizando su Lógica Interna desde la Praxiología Motriz, se presenta como un lapso, así como una posibilidad de contribuir significativamente con el conocimiento sistematizado acerca de la modalidad.

El Fútbol es practicado en los más variados locales y condicionado por diversas formas de organización del juego (Barbosa \& Carvalho, 2008). Más allá de la práctica lúdica realizada en el cotidiano, es fuente de relevantes estudios en el contexto de la investigación científica, que se proyecta de la iniciación deportiva hasta el alto rendimiento (Garganta, Guilherme, Barreira, Brito \& Rebelo, 2013; Gau, 2013; Manoel Sérgio, 2011; Martín-Nebreda, Gutiérrez, Alonso \& García, 2015; Potrac, Jones \& Armour, 2002; Scaglia, 2011). El juego es constituido por un ambiente caótico, cuya variabilidad e imprevisibilidad ganan notabilidad en cuanto elementos dinámicos (Garganta et al., 2013; Grèhaigne; Godbout \& Zeray, 2011). La elevada complejidad del Fútbol da espacio a distintas caracterizaciones del deporte, aspectos esos que sirven como base para el trabajo pedagógico con la modalidad (Jiménez \& Gorostiaga, 2015).

Mucho del conocimiento producido sobre el Fútbol está direccionado al aspecto metodológico. Están relacionados con los distintos contextos (las escuelas, los clubes, las escuelitas), se constituye en cuanto tema de debate y es representado por distintas concepciones (Bettega, Scaglia, Morato \& Galatti, 2015; Casarin, Reverdito, Greboggy, Afonso \& Scaglia, 2011; Scaglia, 2015; Scaglia, Reverdito, Leonardo \& Lizana, 2013). Aún hay indicios de que los distintos contextos prácticos donde se trabaja con la pedagogía vinculada al Fútbol están alejados de las bases teóricas que están siendo producidas (Saad, 2002). Es común que las clases y los entrenamientos sean basados en las experiencias del propio profesor o entrenador en cuanto practicante de la modalidad, así como los criterios que son utilizados para la selección de los contenidos están saturados de componentes culturales del sentido común, sin mayor base científica (Coutinho \& Silva, 2009; Machado, Thiengo \& Scaglia, 2017).

Ante lo expuesto, el estudio se justifica en la medida en que busca, desde la Praxiología Motriz, la construcción de un soporte para el mejor entendimiento de las condiciones de juego en el Fútbol y la indicación de implicaciones a su aspecto pedagógico. En este trabajo, se ha aplicado el término enseñanzaaprendizaje-entrenamiento (Greco, 1998) para referirnos a este proceso. Este concepto se ha utilizado en una serie de estudios con la modalidad (Filgueira y Greco, 2008; Souza, Müller, Costa y Graça, 2014) y englobará aspectos educativos, así como aquellos destinados al rendimiento deportivo, puesto que un proceso de enseñanza y entrenamiento organizados propende a colaborar al desarrollo del aprendizaje de los educandos y los atletas.

La Praxiología Motriz no se propone a ser un método de enseñanza-aprendizaje-entrenamiento, sino un conocimiento científico de los juegos y deportes que permite desvelar su Lógica Interna, es importante señalar (Fagundes \& Ribas, 2017). Buscamos establecer un avance en la comprensión de que se está enseñando, proceso ese que obtendrá mayor consistencia y sentido, ocasionando una mejor organización y selección de contenidos (Ribas, 2005). De este modo, pretendemos impactar en el contexto práctico de los profesores y entrenadores y contribuir para la elección coherente de los métodos de desarrollo del proceso de enseñanzaaprendizaje-entrenamiento. 


\section{Metodología}

El estudio, de carácter teórico, se constituye en cuanto un análisis praxiológico (Fagundes \& Ribas, 2017; Gil, 2017; Marques Filho et al., 2017), bajo una perspectiva cualitativa, decurrente de la necesidad de comprensión, explotación y especificación de determinado fenómeno social (Brito \& Leonardos, 2001; Creswell, 2010), en este caso, el Fútbol. El proceso se caracteriza como descriptivo-propositivo, basado en los criterios indicados por la Praxiología Motriz (Rodríguez Ribas, 2015; Saraví, 2016).

El instrumento de análisis es fundamentado en los Universales de los juegos deportivos colectivos, que son "modelos operativos que representan las estructuras básicas de funcionamiento de todo juego deportivo y contienen su Lógica Interna" (Parlebas, 2001, 463). La estructuración exhibe siete modelos operativos e intenta representar la estructura de funcionamiento de determinada modalidad, lo que posibilita el entendimiento de su Lógica Interna (Lagardera Otero \& Lavega Burgués, 2003; Marques Filho et al., 2017; Parlebas, 2001). Cada modelo es planificado a partir de los siguientes criterios:

\section{Red de Comunicación Motriz}

Se refiere a las interacciones motrices instrumentales entre los participantes, caracterizando como fenómeno mayor en la tarea (Ribas, 2014). De acuerdo con Parlebas (2001), la naturaleza social del juego está fundamentada en la red de comunicación que cualquier juego incluye. Parlebas (1976) resalta la dependencia directa entre el desempeño y la comunicación de los jugadores, siendo que la conducta de cada jugador es siempre comunicativa. Ellos no pueden decidir cuándo iniciar o terminar la comunicación, porque todo el comportamiento motor funciona como una señal (Jiménez \& Gorostiaga, 2015). Pueden caracterizarse en dos tipos: las situaciones de comunicación y la contra-comunicación motriz, establecidas a través de mensajes transmitidos a los compañeros y a los adversarios (Lagardera Otero \& Lavega Burgués, 2003). Cada jugador es portador de mensajes y éstos deben ser transmitidos de modo lo más claro posible a los compañeros y, simultáneamente, difíciles de ser decodificados por los adversarios (Ribas, 2014).

\section{Red de Interacción de Marca}

Centralizará el análisis sobre las maneras de puntuar en el juego, posibilitando ser cooperativa o antagónica (contraria) (Parlebas, 2001). En el Fútbol, se clasifica como una red contraria, ya que el objetivo es hacer el gol en el arco que está siendo defendido por el equipo adversario, además de defender el propio arco.

\section{Sistema de Puntuación}

Corresponde a la manera con que el puntaje es organizado en el sentido de ganar o perder un juego (Parlebas, 2001). Puede estar atada al tiempo de juego, con una duración pre-determinada, a la conquista de un determinado número de puntos, así como un mixto de ambos.

\section{Código Gestémico}

Son posturas y gestos convencionales que transmiten una pretensión o indicación. Es la categoría de actitudes, mímicas, gestos y comportamientos motores puestos en práctica para transmitir pregunta, indicación u orden táctica y relacional, sustituyendo las palabras (Parlebas, 2001). Se refieren a las formas de comunicación gestual facilitadoras de las acciones de relación entre los jugadores.

\section{Código Praxémico}

Considerados como comportamientos de "pre-acción", con un mensaje táctico o relacional (Lagardera Otero \& Lavega Burgués, 2003). Se relacionan a la conducta motriz que es interpretada como un signo, constituyéndose en el propio comportamiento observable de un jugador (Parlebas, 2001). Según Sampedro Molinuevo (1996), se puede definir como acciones que tienen como significado o intencionalidad de ser un mensaje previo de la ejecución de una interacción motriz.

\section{Sistema de Cambio de Roles}

Permite desvelar las distintas actuaciones adoptadas por los jugadores y los cambios posibles entre ellas. Son "clases de comportamientos motores asociadas a un reglamento preciso, en un juego deportivo. Toda actuación socio-motor está asociada a un estatuto que codifica la práctica” (Parlebas, 2001, 132). La 
especificación de las actuaciones puede ser realizada siguiendo distintos criterios. En los juegos deportivos colectivos, frecuentemente se utiliza el criterio desde la posesión de pelota. Así, las actuaciones se caracterizan por: el jugador con pelota, el jugador sin pelota del equipo que detiene la posesión y el jugador del equipo que no la detiene (Hernandéz Moreno, 2000).

\section{Sistema de Cambio de Subroles}

Abarca las Acciones Motrices posibles de ser efectuadas por el jugador de acuerdo con su interpretación. No obstante, los jugadores con el mismo role socio-motor disponen de la misma amplitud de Acciones Motrices como la posibilidad de utilización. Representan la secuencia motriz de un jugador, considerada como una unidad básica de comportamiento estratégico (Lagardera Otero \& Lavega Burgués, 2003). Por ser desarrollados desde los roles, son más numerosos e indican las maneras directas del participante proceder en el juego (Lavega Burgués, 2008).

\section{Resultados y Discusión}

Los resultados se organizan a partir de cada uno de los Universales de los juegos deportivos colectivos para que, así, se pueda relacionar el contexto del fútbol con estos elementos de la Praxiología Motriz. Apoyado en esta estructuración, son expuestas las influencias y posibilidades del proceso de enseñanza-aprendizajeentrenamiento del deporte.

\section{Red de Comunicación Motriz}

Posibilita analizar la estructura comunicacional en el contexto de cooperación y oposición del Fútbol. Un buen desempeño, individual y colectivo, depende directamente de la comunicación de los jugadores, ya que la conducta de cada jugador es siempre comunicativa (Parlebas, 1976). En este sentido, todas las Acciones Motrices que son ejecutadas en el juego están delimitadas por las interacciones de cooperación y oposición existentes en él (Jiménez \& Gorostiaga, 2015).

La Lógica Interna del Fútbol aporta un ambiente de gran variabilidad de acontecimientos. Hay el enfrentamiento de dos equipos que buscan equilibrarse tácticamente y generar el desequilibrio en el equipo adversario (Garganta, 1997). Se constituye un contexto dinámico y complejo, en el cual emergen posibilidades de acción a todo el momento (Bettega et al., 2016; Garganta \& Cunha y Silva, 2000). El expresivo número de interacciones establecidas entre los participantes corrobora para un alto grado de complejidad del juego, en un panorama en el cual 22 jugadores buscan arreglos comportamentales constantes con el objetivo de potenciar las acciones cooperativas y contrarias (Fernandes Malta, 2012; Novaes, Rigon $\&$ Dantas, 2014), como se ve en la Figura 1:

Figura 1. Red de Comunicación Motriz del Fútbol.

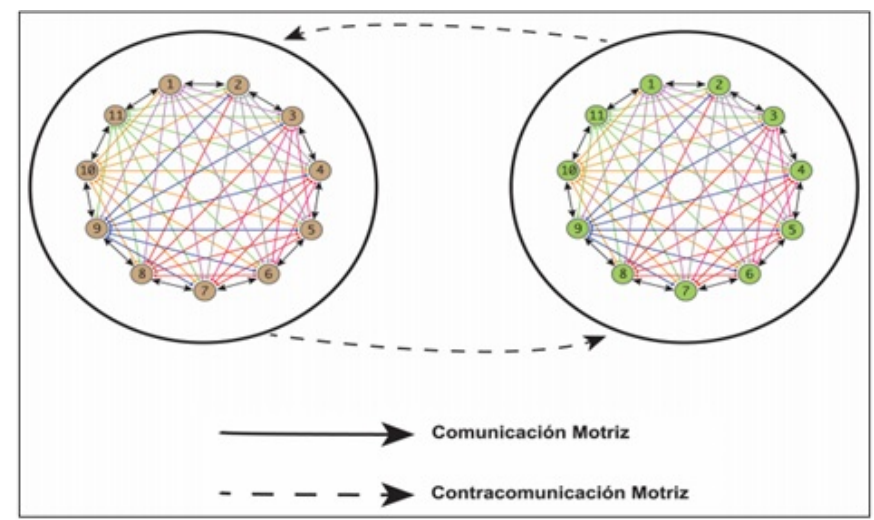

Fuente: Adaptada de Díaz (2015) 
La lectura de juego y la interacción con los demás participantes y con la pelota se sitúan como elementos centrales para una performance de cualidad. Las capacidades tácticas de los jugadores y sus procesos cognitivos subyacentes a la toma de decisión se constituyen como requisitos esenciales a la excelencia del desempeño deportivo (Mcpherson, 1994). En el transcurrir de un partido surgen inumerables situaciones cuya intensidad, orden cronológico y frecuencia no pueden ser previstas, exigiendo una elevada capacidad de adaptación y de respuesta inmediata por parte de los jugadores y de los equipos, acarreando que los jugadores tomen decisiones bajo condiciones de inseguridad (Garganta, 1997; Jiménez \& Gorostiaga, 2015). Por lo tanto, la eficiencia de las Acciones Motrices de cada jugador está atada a su adaptabilidad y su capacidad de anticipación.

$\mathrm{Al}$ atribuir elevada centralidad a los elementos puramente técnicos del juego, no son contempladas las demandas de la Lógica Interna del mismo. Ejecuciones motoras correctas o gestos técnicos, ideas, solo existen en la especificidad de resolver los problemas de la situación motriz que es expresada en determinado momento (Garganta, 1997; Greco, 1998; Parlebas, 1999). Como las Acciones Motrices del Fútbol solo existen si son relacionadas con su Red de Comunicación, aspectos técnicos y tácticos se caracterizan como indisociables en todos los momentos del juego.

La Red de Comunicación será uno de los Universales esenciales para orientar el trabajo del entrenador. De acuerdo con Saad (2002), en la mayoría de los programas de entrenamientos existe la preferencia por metodologías directivas, en las cuales ocurren muchas restricciones a entrenos técnicos. Martin, Carl \& Lehnert (2008) indican que la enseñanza del Fútbol es un proceso complejo, dirigido al desarrollo planeado de determinadas condiciones de desempeño deportivo. En este sentido, con la comprensión del carácter interaccionista del Fútbol y del concepto de la Acción Motriz, el proceso de enseñanza-aprendizajeentrenamiento será coherente con la Lógica Interna del juego. Para construirse, con lo entrenamiento, los conocimientos realmente útiles a la especificidad del Fútbol, todo ese proceso debe contener, por principio básico, la idea de que todo lo que ocurre en el juego está condicionado por las interacciones presentes en él.

\section{Red de Interacción De Marca}

La Red de Interacción de Marca del Fútbol se clasifica como antagónica. Significa que, aunque el juego también contenga cooperación, la forma de puntuar sucede por medio de oposición. Según Amiero (2010), un equipo debe generar desequilibrios en el sistema defensivo adversario, creando espacios con el objetivo de construir situaciones para finalizar al gol. Cuando comparado con juegos deportivos colectivos, el Fútbol presenta una supremacía de la defensa sobre el ataque (Bauer \& Ueberle, 1988; Dufour, 1989). Por eso, una de las grandes dificultades en el juego de Fútbol es conseguir crear oportunidades de finalización (Castelo, 1994).

La relación de ataque y defensa del juego del Fútbol recibe valía. Bayer (1994) propone principios operativos que caracterizan esas etapas, siendo los ofensivos: la conservación individual y la colectiva de la pelota, progresión al blanco adversario y finalización de la jugada; y los defensivos: recuperación de la pelota, impedir la progresión del equipo adversario y protección del blanco. Desde estos principios, emergen reglas de acción para su operacionalización (Daolio, 2002). Estos elementos formarán la estructura del proceso, cooperativo y contrario, que constituye la Red de Interacción de Marca, buscando en definitivo el aspecto contrario, de superar la defensa adversaria a fin de de marcar el gol.

En el contexto de las clases y los entrenamientos, las actividades situacionales que incorporan etapas de finalización del juego necesitan de la presencia de la oposición como esencia. La colocación de accesorios inertes que representan un adversario, tales como objetos en lugar del arquero, abstraen el elemento central que caracteriza la Red de Interacción de Marca del Fútbol (Amaral, 2004; Parlebas, 2001). 
Luego, es primordial contemplar el aspecto contrario, que puede partir desde su unidad más básica, que fue caracterizada por el $1 \times 1$, hasta una propuesta que involucra un mayor número de participantes e interacciones.

\section{Sistema de Puntuación}

A priori, el Sistema de Puntación es bastante sencillo y de común entendimiento por la mayor parte del público que se interesa por Fútbol. La contabilización de los puntos es realizada a través de los goles que son hechos y la finalización del partido que ocurre al alcanzar el tiempo límite. Entretanto, el Sistema de Puntaje también tendrá una relación dinámica de influencia en las situaciones motrices del juego. Un equipo, al entrar en ventaja o desventaja en el marcador, podrá alterar los aspectos tácticos y estratégicos con el objetivo de optimizar el mantenimiento de la victoria o revertir el marcador adverso (Armatas, Yiannakos, Zaggelidis, Papadopoulou \& Fragkos, 2009; Pujals \& Fiorese, 2002; Vargas, Saretti \& Bojikian, 2011). Considerando que la ocurrencia de un gol puede ser un elemento de referencia para la adecuación de la organización colectiva, percibimos que el Sistema de Puntaje tendrá relación con las Acciones Motrices, ya que influenciará en las interacciones cooperativas y contrarias del juego.

Evidenciamos la importancia de considerar el Sistema de Puntaje al pensarse en el proceso de enseñanzaaprendizaje-entrenamiento. Este indicativo indica para la sistematización de actividades que contemplen situaciones-problema desde la superioridad y la inferioridad en el marcador, así como las relacionadas al tiempo de juego. Desde esta propuesta, agregamos una mayor variedad de elementos que son trabajados en los entrenamientos, enriqueciendo el desarrollo de los participantes desde constreñimientos ajustados con la Lógica Interna del juego.

\section{Código Gestémico}

Mientras la Red de Comunicación aborda las interacciones comunicacionales directas del juego, el Código Gestémico irá a abarcar una forma de comunicación indirecta. Presentamos, como ejemplo, un jugador levantar el brazo para pedir un pase o indicar con la mano la trayectoria que irá a seguir. Los gestemas pueden ser pasibles de lectura solamente por compañeros o también por adversarios, denominados particulares y unívocos, respectivamente (Parlebas, 2001). De esta manera, se constituyen en cuanto facilitadores a la transmisión de mensajes entre los participantes. Son muy utilizados en la etapa de iniciación, mientras tanto, en el alto rendimiento ocurren de forma menos cotidianas. La facilidad de lectura por parte del adversario explica su menor incidencia en contextos de desempeño elevado (Ribas, 2014). En niveles iniciales, con practicantes menos eficientes, favorecerá el intercambio de informaciones entre compañeros, con perjuicio amenizado debido a las capacidades de lectura de juego todavía primarias de los adversarios (Lagardera Otero \& Lavega Burgués, 2003).

Por este motivo, el maestro debe permitir que los gestemas se utilicen en un contexto de iniciación, con el fin de perfeccionar la comunicación y la consecuente organización colectiva. Sin embargo, es importante evitar la dependencia de este recurso, progresando a lecturas en el ámbito praxémico, como se discute en el ítem a continuación. Además, los gestemas se pueden usar a partir de una combinación previa entre compañeros de equipo, destinadas a momentos específicos del juego, lo que dificulta la lectura del oponente. Como ejemplos, podemos mencionar las jugadas ensayadas, comúnmente señaladas por el ejecutor de tiro libre.

\section{Código Praxémico}

Los praxemas están directamente relacionadas con el proceso de toma de decisión, pues son indicadores de la Acción Motriz consiguiente. Según Ericson (2003), los jugadores peritos son mejores en la captura temprana de indicadores relevantes de la tarea. Por lo tanto, una lectura praxémica perfeccionada permite, por parte del atleta, una adecuada orientación para los indicadores más relevantes, utilizando determinadas 
informaciones que emergen relativamente tempranas en el escenario y que, veían relaciones probabilísticas, permiten predecir el resultado de la acción (Ericson, 2003). Los jugadores deben tener la capacidad de comprender el significado táctico de los movimientos de los otros jugadores (los compañeros y los adversarios), o sea, aprender a descifrar los praxemas en el juego. El proceso de enseñanza-aprendizajeentrenamiento de esas capacidades es largo y complejo, exigiendo una serie de acciones didácticas, como modificaciones del juego para proporcionar el desarrollo de esas competencias (Jiménez \& Gorostiaga, 2015). Como ejemplos, hay actividades que no solo obligan a la reacción sobre los movimientos de los compañeros y oponentes, sino a la anticipación en relación a los mismos, colaborando para el desarrollo de la aptitud de lectura praxémica de los atletas.

Un praxema, que dispone la propiedad inhabitual de ser parte integrante de una acción, es la unidad semiótica de los procesos cognitivos y comunicativos que ocurren en un juego de Fútbol (Parlebas, 2001). Es la acción en sí, pero con significados distintos de acuerdo con quien la está produciendo y, por consiguiente, los jugadores con más habilidad para descifrar praxemas tendrán mejor desempeño en una determinada situación de juego (Jiménez \& Gorostiaga, 2015). Las lecturas praxémicas se darán a todo instante entre los jugadores que ocupan el mismo rol en el momento (jugador con la pelota, jugador sin la pelota del equipo que detiene la posesión y jugador del equipo que no detiene la posesión). Esas lecturas también serán realizadas entre los distintos roles, siendo cooperativas o contrarias. La Figura 2 ilustra este concepto:

Figura 2. Lecturas Praxémicas del Fútbol.

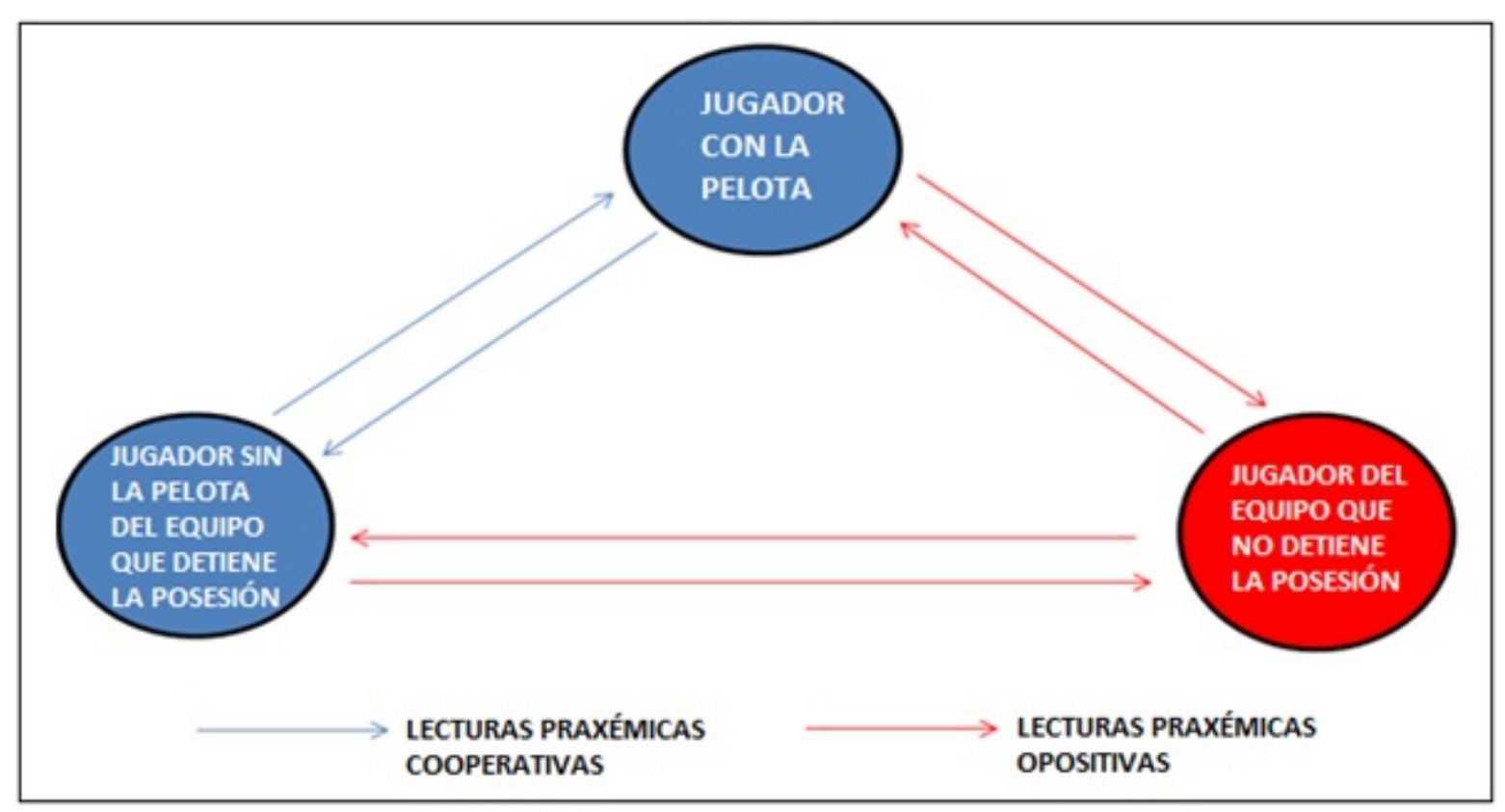




\section{Sistemas de Cambio de Roles y de Subroles}

De acuerdo con el rol que el jugador asume durante el juego de Fútbol, surgen las posibilidades de Acciones Motrices a ser realizadas (Subroles), al mismo paso que las relaciones de interacción entre los jugadores influenciarán en su utilización. Al pensar los subroles presentes en el Fútbol, es común percibir la mayor atención dada a aquellos realizados con pelota, como: el tiro, el pase, la finta, el cabeceo, la tirada, la protección de la pelota y lanzamientos laterales (Leal, 2000; Mahlo, 1969; Mutti, 2003; Santos, 1979). Los subroles de los jugadores sin la pelota del equipo que detiene la posesión y de los jugadores del equipo que no detiene la posesión son, muchas veces, descuidados.

Desde la comprensión de los roles existentes en el juego y de sus respectivos subroles, es posible estructurar todas las Acciones Motrices del Fútbol, balizadas por los criterios de la Praxiología Motriz, de acuerdo con lo presentado en la Tabla 1. De esta manera, organizamos una base de conocimiento que contribuye con trabajo pedagógico en el Fútbol, cooperando para la sistematización de contenidos y la escogencia de métodos coherentes para el desarrollo de los mismos por parte de los entrenadores. Es esencial recalcar las particularidades cuanto a las Acciones Motrices del arquero, ya que él puede utilizar sus miembros superiores, de acuerdo con lo previsto por la regla. En esta investigación, abordamos solamente las Acciones Motrices relativas a los jugadores de línea, enfocando el estudio de las especificidades del arquero en cuanto demanda para investigación posterior.

Tabla 1. Acciones Motrices del Fútbol

\begin{tabular}{|l|l|l|}
\hline Jugador con la pelota & $\begin{array}{l}\text { Jugador sin la pelota del } \\
\text { equipo que detiene la } \\
\text { posesión }\end{array}$ & $\begin{array}{l}\text { Jugador del equipo que no } \\
\text { detiene la posesión }\end{array}$ \\
\hline Dominar & Posicionarse & Desmarcarse \\
Conducir & Desplazarse & Posicionarse \\
Proteger & Fintar & Marcar el adversario \\
Pasar & & Anticiparse (buscar \\
Finalizar al gol & interceptar la pelota) \\
Alejar & & \\
\hline
\end{tabular}

Fuente: elaborada por los autores.

Realzamos que todas esas Acciones Motrices que fueron mencionadas ocurren dentro de un contexto de cooperación y oposición, por esta razón, siempre están enlazadas con esos dos elementos, siendo influenciadas por ellos e influenciándolos. No obstante, cada Acción Motriz detiene un aspecto que prepondera en el sentido dado a ella (cooperar u oponerse). Esa predominancia no descarta la otra forma de interacción presente en la acción, mientras tanto, revela cual es mayoritaria en el sentido dado de la Acción Motriz. Todavía hay acciones sin predominancia, en que ni la cooperación ni la oposición se sobresale una sobre la otra. Las Acciones Motrices del Fútbol que son caracterizadas desde un criterio de predominancia cuanto a la cooperación y oposición en los tres roles son ilustradas didácticamente en las figuras 3, 4 y 5. 
Figura 3. Predominancia de las Acciones Motrices del jugador con la pelota.

\section{JUGADOR CON LA PELOTA}
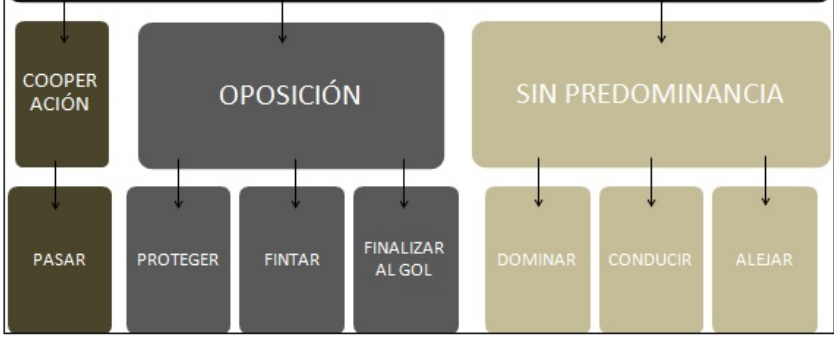

Fuente: elaborada por los autores.

Figura 4. Predominancia de las Acciones Motrices del jugador sin la pelota del equipo que detiene la posesión.

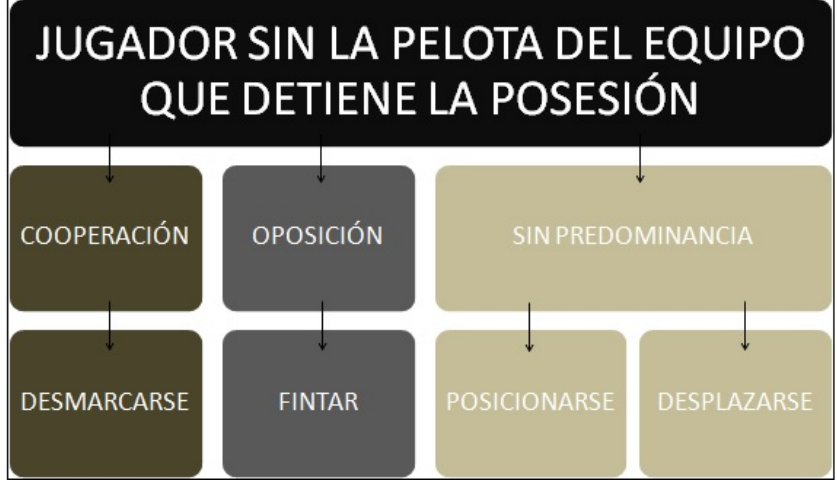

Fuente: elaborada por los autores

Figura 5. Predominancia de las Acciones Motrices del jugador del equipo que no detiene la posesión.

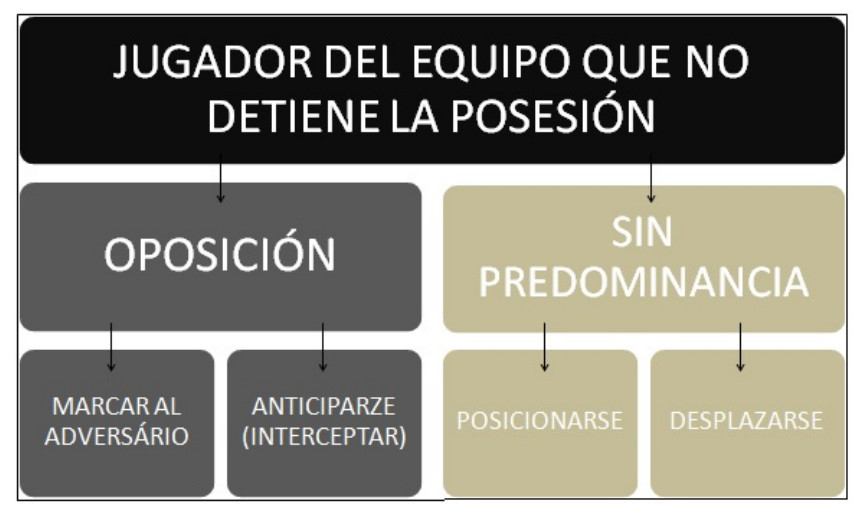

Fuente: elaborada por los autores. 
Tales conocimientos serán de suma importancia para que el profesor o el entrenador refleja sobre sus métodos de enseñanza-aprendizaje-entrenamiento. Es frecuente entrenar algunas Acciones Motrices que son descontextualizadas de sus reales características de interacciones. ¿De qué sirve entrenar el pase utilizando conos como blancos, si la acción de pasar está predominantemente atada a la cooperación? ¿De qué sirve trabajar la finalización al gol sin la presencia del adversario, si será por medio de la oposición que esa acción será caracterizada en un contexto de juego? Dudas como esas son respondidas a través de esta criteriosa mirada a respecto de la Lógica Interna del juego, proporcionado por la Praxiología Motriz. Al cuantificarse las Acciones Motrices considerando el criterio delimitador de las interacciones que son establecidas en el juego y los roles que son ejercidos por los jugadores, se convierte posible obtener otros datos que pueden aportar con el planeamiento y la organización de las actividades que son propuestas por el entrenador para sus entrenamientos. Tales sistematizaciones están expuestas en las Figuras 6 y 7.

Figura 6. Acciones Motrices y las interacciones.

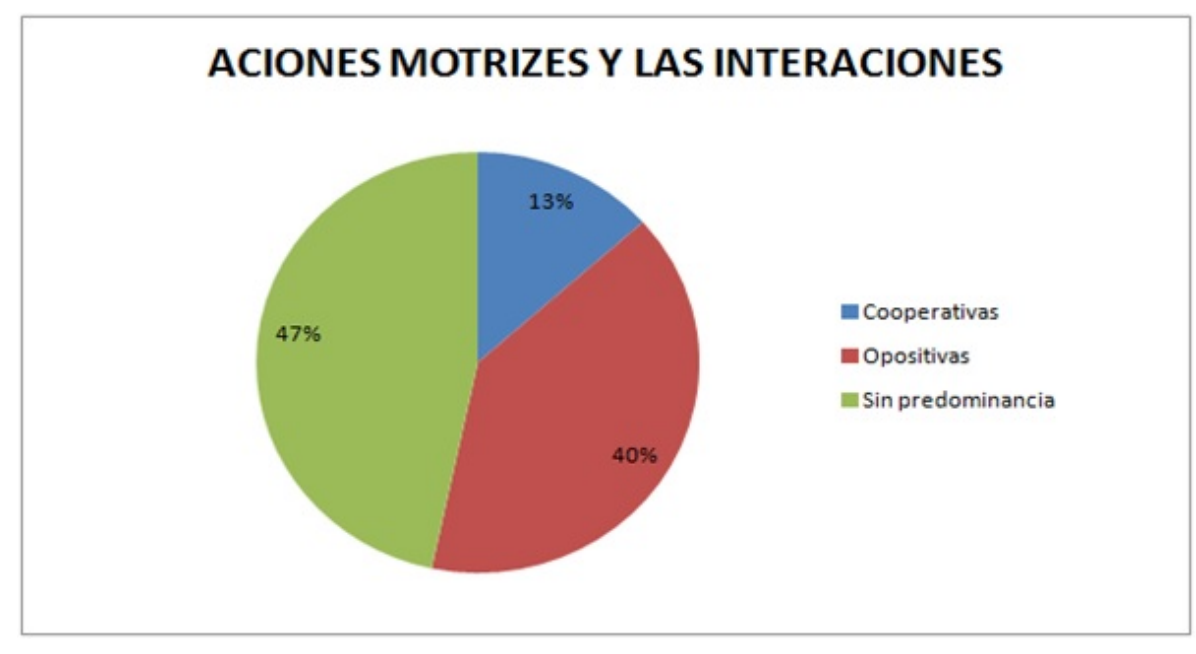

Fuente: elaborada por los autores

Percibimos que poco más del 13\% de las Acciones Motrices es predominantemente cooperativa. Eso genera una reflexión sobre el tiempo malgasto con las actividades que contemplen solamente la cooperación. Ejercicios que utilizan dos jugadores uno delante el otro, cambiando pases de las más variadas formas, son mucho usuales en el contexto de los entrenamientos. Con todo, la ausencia de oposición denota la incoherencia con las características de la Lógica Interna del Fútbol. Al profundizar el entendimiento de las Acciones Motrices que están relacionadas con las interacciones, convertimos posible constatar que la preferencia por ciertos tipos de actividades puede no estar en sincronía con las demandas existentes en el contexto del juego.

Al discurrir el desarrollo de las Acciones Motrices de los atletas, debemos direccionar mayor atención para la forma con que ellos actuarán en el juego, desde los roles que ejercerán (Ribas, 2014). La dinámica de cambio de roles hará con que, a todo momento y simultáneamente, los subroles también cambien. Así, al pensar la variedad de las posibilidades de actuar en el Fútbol, Acciones Motrices se distribuyen del siguiente aspecto: 
Figura 7. Acciones Motrices y los roles.

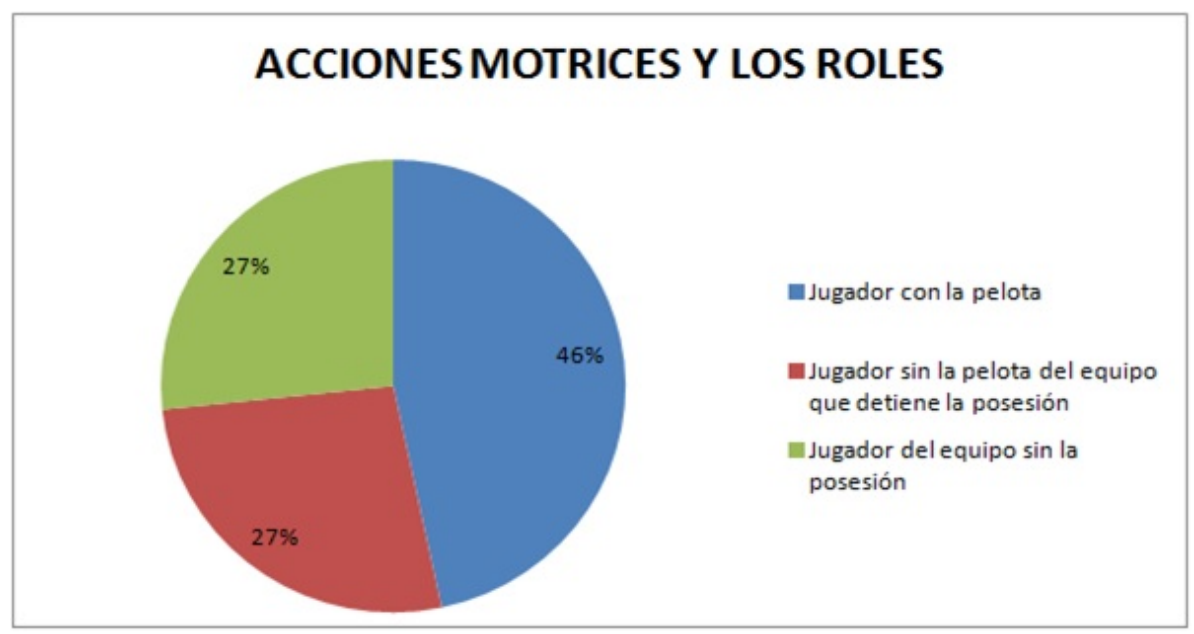

Fuente: elaborada por los autores

Percibimos que, de entre los roles, las posibilidades de Acciones Motrices (subroles) del jugador con la pelota son mayoría, lo que representa casi la mitad de la totalidad actuante en el juego. Así, en un juego que cuenta con 22 jugadores y solamente una pelota, la variedad de situaciones y la variedad de Acciones Motrices que son trabajadas pueden ser reducidas si un jugador tener muy poco contacto con la pelota. En ese contexto, actividades con el campo reducido o el número menor de participantes harán con que los jugadores tengan mayor contacto con la pelota, asumiendo el rol de jugador con la pelota más veces. Según Sadi, Costa y Sacco (2008), ese tipo de actividad trabaja directamente con las tomas de decisión, así como selección y ejecución de habilidades con la pelota. Así, dando la oportunidad al practicante, la posibilidad de disponer de una estructura mayor de subroles que están conectados directamente a las lecturas de juego.

El conocimiento de la distribución de las Acciones Motrices en relación a los roles además proporciona una atención distintiva a las acciones de los jugadores sin la pelota. Muchas veces la preocupación en el proceso de enseñanza-aprendizaje-entrenamiento está centrada en el jugador con la pelota. De esta suerte, se deja de dar la debida consideración a la gran parte de las Acciones Motrices posibles en el juego, que son realizadas por los jugadores que no detienen la pelota. Conforme Costa, Garganta, Greco y Mesquita (2010) el jugador pasa en promedio el 97\% del tiempo de juego sin la posesión de la pelota. De esa manera, el desarrollo de las Acciones Motrices que son realizadas sin la pelota debe ser contemplado en el proceso de enseñanzaaprendizaje-entrenamiento.

\section{ConcLusiones}

La complejidad existente en el Fútbol permite múltiples entendimientos sobre el juego e influencia en el trabajo pedagógico con la modalidad. El uso de la Praxiología Motriz se justifica porque nos proporciona una base para que los maestros y entrenadores desarrollen un conocimiento profundo sobre el Fútbol, y también permite proponer aspectos indicativos del aspecto metodológico del proceso de enseñanza-aprendizajeentrenamiento. A través de los Universales de los juegos deportivos colectivos, podemos concebir principios de enseñanza que respeten las demandas del juego. 
La comprensión de la Red de Comunicación nos permite comprender las condiciones del juego en función de las interacciones dentro de él. Se destaca el aspecto comunicativo, que guía los criterios para adoptar ciertos métodos de enseñanza-aprendizaje-entrenamiento en detrimento de otros. El proceso de fragmentar el juego en partes que excluyen su naturaleza cooperativa y opositora deja de tener sentido, dando paso a una perspectiva basada en la Lógica Interna que considera el Fútbol en su totalidad. La Red de Interacción d Marca y el Sistema de Puntuación permiten comprender las demandas dirigidas al objetivo del juego (hacer goles y no permitir que el oponente los haga) de una manera amplia y compleja. Tales elementos llegan a ser vistos como esenciales, conformando la organización pedagógica de maestros y entrenadores de manera coherente.

Asimismo, la mirada sobre la interacción entre los participantes también se profundiza sobre la comunicación indirecta, en la que tenemos los gestemas y praxemas, presentados desde su potencial al proceso de enseñanza-aprendizaje-entrenamiento. Los elementos relacionados con jugar con y sin la pelota, así como las posibilidades de acción para los otros roles que se pueden adoptar en el juego, muestran una mayor pluralidad de información sobre las situaciones de manejo expresadas en el juego. De esta manera, nos damos cuenta de que el rendimiento del atleta estará fusionado a su adaptabilidad a los diferentes sub-roles que se puede efectuar durante el juego.

Este contexto nos lleva a la comprensión de las múltiples Acciones Motrices y la organización de sus características principales, componiendo una comprensión necesaria para suministrar su desarrollo calificado por los atletas. La presentación de cada una de estas acciones, la relación con su esencia cooperativa o opositora y su surgimiento de los diferentes roles y sub-roles, generan recursos que contribuyen a asegurar un trabajo pedagógico llevado a cabo con coherencia, independientemente de su contexto práctico y sus propósitos. En otras palabras, la base para comprender la Lógica Interna del Fútbol perdura y, a partir de ahí, los maestros y entrenadores podrán dirigir el proceso de enseñanza-aprendizaje-entrenamiento hacia sus objetivos, ya sea relacionado con el deporte recreativo, participación o alto rendimiento, tanto para niños como para adultos.

Es primordial enfatizar que esta investigación no contempla todas las posibilidades existentes sobre la enseñanza-aprendizaje-entrenamiento del Fútbol. Se caracteriza por ser un primer movimiento que busca relacionar el conocimiento de la Praxiología Motriz con el Fútbol. La aproximación de este marco teórico con la intervención en contextos prácticos se manifiesta como una posibilidad interesante para la secuencia del desarrollo de estas discusiones.

\section{Agradecimientos}

El presente trabajo fue realizado con el apoyo de la Coordinación de Perfeccionamiento de Personal de Nivel Superior - Brasil (CAPES) - Código de Financiación 001.

Agradecemos al Espaço da Escrita (Vicerrectorado de Investigación - Unicamp) por la traducción de este artículo al español.

\section{REFERENCIAS}

Amaral, R. M. (2004). A Modelação do Jogo em Futsal: Análise Sequencial do $1 x 1$ no Processo Ofensivo. 179 f. Mestrado em Ciência do Desporto, Faculdade de Ciências do Desporto e de Educação Física, Universidade do Porto.

Amiero, N. (2010). A Defesa Zona no Futebol: Um Pretexto para Refletir Sobre o "Jogar" Bem, Ganhando. Lisboa: Visão e Contextos.

Armatas, V.; Yiannakos, A., Zaggelidis, G.; Papadopoulou, S., \& Fragkos, N. (2009) Goal scoring patterns in Greek top leveled soccer matches. Journal of Physical Education and Sport, 23(2), 1-5. Recuperado de https://www.researchgate.net/publication/26627711_GOAL_SCORING_PATTERNS_IN_GREEK_T OP_LEVELED_SOCCER_MATCHES 
Barbosa, B. T. C., y Carvalho, A. M. (2008) Incidência De Lesões Traumato-Ortopédicas Na Equipe Do Ipatinga Futebol Clube-MG. Movimentum - Revista Digital de Educaçáo Física, 3(1). Recuperado de https://www.unilestemg.br/movimentum/Artigos_V3N1_em_pdf/movimentum_v3_n1_barbosa_bruno _teixeira_casoti_2_2007.pdf

Bauer, G., y Ueberle, H. (1998) Fútbol: Factores de Rendimiento, Dirección de Juegadores y del Equipo. Barcelona: Martinez Roca.

Bayer, C. (1994). O ensino dos deportos colectivos. Lisboa, Dinalivro.

Bettega, O. B.; Galatti, L. R.; Schmitz Filho, A. G.; Tozetto, A. B.; Longarela, B., y Scaglia, A. J. (2016). Planificación Táctica en el Fútbol: Aspectos Generales y Específicos. E-Balonmano.com: Revista Ciencias del Deporte, 12(1), 45-52. Recuperado de http://www.e-balonmano.com/ojs/index.php/revista/article/view/309/v12-n1-a3

Bettega, O. B.; Scaglia, A. J.; Morato, M. P., y Galatti, L. R. (2015) Formação de Jogadores de Futebol: Princípios e Pressupostos para Composição de Uma Proposta Pedagógica. Movimento, 21(3), 791-801. doi: https://doi.org /10.22456/1982-8918.49051

Brito, A. X. y Leonardos, A. C. (2001) A identidade das Pesquisas Qualitativas: Construção de Um Quadro Analítico. Cadernos de Pesquisa, 31(113), 7-38. Recuperado de http://www.scielo.br/pdf/cp/n113/a01n113.pdf

Casarin, R. V.; Reverdito, R. S.; Greboggy, D. L.; Afonso, C. A., y Scaglia, A. J. (2011).Modelo de Jogo e Processo de Ensino no Futebol: Princípios Globais e Específicos. Movimento, 17(3), 133-152. doi: https://doi.org/10.2245 6/1982-8918.16302

Castelo, J. (1994) Futebol, Modelo Técnico-Tático do Jogo. Lisboa: Edições FMH.

Costa, I. T.; Garganta, J.; Greco, P. J., y Mesquita, I. (2010). Análise E Avaliação Do Comportamento Tático No Futebol. Revista da Educação Física, 21(3), 443-455. doi: https.//doi.org./10.4025/reveducfis.v21i3.8515.

Coutinho, N. F. y Silva, S. P. S. (2009). Conhecimento e Aplicação de Métodos de Ensino para os Jogos Esportivos Coletivos na Formação Profissional em Educação Física. Movimento, 15(1), 117-144. https://doi.org/10.2245 6/1982-8918.2086

Creswell, J. W.. (2010) Projeto de pesquisa: métodos qualitativo, quantitativo e misto (3 ed.) Porto Alegre: Artmed.

Daolio, J. (2002). Jogos Esportivos Coletivos: dos Princípios Operacionais aos Gestos Técnicos - Modelo Pendular a Partir das Idéias de Claude Bayer. Rev. Bras. Ciênc. e Mov., 10(4), 99-104. http://dx.doi.org/10.18511/rbcm .v10i4.478

Díaz, R. D. (2015). Análisis Praxiológia de la Dinámica de Juego em Fútbol: Lógica Externa y Lógica Interna. 437 f. Tese (Doutorado em Praxiologia Motriz, Educação Física e Treinamento Desportivo) - Departamento de Educación Física, Universidad de Las Palmas de Gran Canaria, Las Palmas de Gran Canaria.

Dufour, W. (1989) Football: Les Techniques D’Observation du Comportement Moteur. EPS Education Physique \& Sport, 217, 68-73.

Ericsson, K. A. (2003). Development of Elite Performance and Deliberate Practice: an Update From the Perspective of the Expert Performance Approach. In: Starkes J., Ericsson K. A. (1 ed.), Expert Performance in Sports Advances in Research on Sport Expertise. Human Kinects.

Fagundes, F. M. y Ribas, J. F. M. (2017). A Dinâmica do Voleibol Sob as Lentes da Praxiologia Motriz: Uma ANálise Praxiológica do Levantamento. Revista Brasileira de Ciência e Movimento, 25(3), 134-149. http://dx.doi.org/1 $0.18511 / \mathrm{rbcm} . v 25 \mathrm{i} 3.7575$

Fernandes Malta, P. G. (2012) Caracterização da Transição Defesa-Ataque de uma Equipe de Futebol. 17 f. Dissertação (Mestrado em Ciências do Desporto) - Universidade da Beira Interior, Covilha.

Filgueira, F. M. y Greco, P.J. (2008) Futebol: um estudo sobre a capacidade tática no processo de ensino-aprendizagemtreinamento. Revista Brasileira de Futebol, 1(2), 53-66. Recuperado de https://rbf.ufv.br/index.php/RBFuteb $\mathrm{ol} /$ article/view/34/32

Garganta, J. (1997) Modelação Táctica do Jogo de Futebol: Estudo da Organização da Fase Ofensiva em Equipas de Alto Rendimento. 318 f. Tese (Doutorado em Educação Física) - Faculdade de Ciências do Desporto e da Educação Física, Universidade do Porto, Porto. 
Garganta, J. y Cunha e Silva, P. (2000). O Jogo de Futebol: Entre o Caos e a Regra. Revista Horizonte, 16 (91), 5-8. Garganta, J.;Guilherme, J.; Barreira, D.; Brito, J., y Rebelo, A. Fundamentos e práticas para o ensino e treino do Futebol. (2013) In: Tavares, F. (Org.). Jogos desportivos coletivos: ensinar a jogar (pp. 199-263). Porto: Editora FADEUP.

Gau, L. (2013). Trends and topics in sports research in the Social Science Citation Index from 1993 to 2008. Perceptual \& Motor Skills, 116(1), 305-314. https://doi.org/10.2466/30.03.PMS.116.1.305-314

Gil, J. S. (2017). Una aproximación a las dificultades del deporte femenino en la alta competición: Hacia la equidad e igualdad. Acción Motriz, 18, 29-36. Recuperado de https://dialnet.unirioja.es/servlet/articulo?codigo=6324715

Greco, P. J. (org.). Iniciação Esportiva Universal. Belo Horizonte: UFMG, 1998.

Gréhaigne, J. F.; Godbout, P., \& Zeray, Z. (2011). How the "rapport de forces" evolves in a soccer match: the dynamics of collective decisions in a complex system. Revista de Psicología del Deporte, 20(2), 747-765. Recuperado de ht tps://www.redalyc.org/articulo.oa?id=235122167032

Hernández Moreno, J. (2000). La Iniciación a los Deportes desde su Estructura y Dinámica - Aplicación a la Educación Fisica Escolar y al Entrenamiento Deportivo. Barcelona: Inde.

Hernández Moreno, J. (2001). Anàlisi dels paràmetres espai i temps en el futbol sala. La distància recorreguda, el ritme i la direcció del desplaçament del jugador durant un partit de competició: Els casos de J. Gay (defensa), C. Marrero (lliure), J. Beto (pivot), J. Limones (aler) i J. Clavería (porter). Apunts. Educación Física i Esports, 65(3), 32-44. Recuperado de https://dialnet.unirioja.es/servlet/articulo?codigo $=150390$

Jiménez, D. M. y Gorostiaga, R. M. S. (2015). Data Quality Control of an Observational Tool to Analyze Football Semiotricity. Cuadernos de Psicología del Deporte, 15(1), 223-232. Recuperado de https://digitum.um.es/digitu $\mathrm{m} /$ bitstream/10201/44320/1/223431-788691-1-SM.pdf

Lagardera Otero, F. y Lavega Burgués, P. (2003) Introducción a la Praxiología Motriz. Barcelona: Paidotribo.

Lavega Burgués, P. (2008). Classificação dos Jogos, Esportes e as Práticas Motrizes. In: Ribas, J. F. M.(Org.). Jogos e esportes: fundamentos e reflexões da Praxiologia Motriz (pp. 50-21). Santa Maria: Editora da UFSM.

Leal, J. C. (2000) Futebol: Arte e Oficio. Rio de Jaineiro: Sprint.

Machado, J. C.; Thiengo, C. R., y Scaglia, A.J. (2017). A Formação do Treinador de Iniciação Esportiva: O que é Preciso Aprender para Ensinar Futebol. In: Galatti, L. R.; Scaglia, A. J.; Montagner, P. C., y Paes, R. R.(orgs.) (1 ed,), Desenvolvimento De Treinadores e Atletas: Pedagogia do Esporte (pp. 163-187). Campinas: Editora Unicamp.

Mahlo, F. (1969). O Acto Táctico no Jogo. Lisboa: Compedium.

Manuel Sérgio. (2011). Filosofia do Futebol. Lisboa: PrimeBooks.

Mancilla, G. H., y Pozo, P. P. (2013) Programa de entrenamiento praxiológico para la técnica de los pases en el fútbol en alumnos de la institución educativa "Corazón de Jesús". 352 f. Tese (Doutorado em Educação Física) - Faculdad de Educación, Universidad Nacional San Cristobal de Humanga, Ayacucho.

Marques Filho, C. V.; Schmitz Filho, A. G.; Ribas, J. F. M., y Bettega, O. B. (2017). O Goleiro de Futebol: Uma Visão a Partir da Praxiologia Motriz. Revista Brasileira de Futsal e Futebol, 9(35), 406-415. Recuperado de http://ww w.rbff.com.br/index.php/rbff/article/view/541/435

Martín Nebreda, X.; Gutiérrez, M. P.; Alonso, M. G., y García, C. G. (2015). Producción científica sobre fútbol en revistas españolas de ciencias de la actividad física y del sciences. Movimento, 21(3), 659-672. https://doi.org/1 $0.22456 / 1982-8918.54313$

Martin, D.; Carl, K., y Lehnert, K. (2008). Manual de Teoria do Treinamento Esportivo. São Paulo: Phorte.

Mcpherson, S. (1994). The Development of Sport Expertise: Mapping the Tactical Domain. Quest, 46(2), 223-400. https://doi.org/10.1080/00336297.1994.10484123

Mutti, D. (2003). Futsal: Da Iniciação ao Alto Nivel. São Paulo: Phorte.

Novaes, R. B., Rigon, T. A., Dantas, L. B. T. (2014). Modelo de jogo de futsal e subsídios para o ensino. Movimento, 20(3), 1039-1060. https://doi.org/10.22456/1982-8918.39355

Parlebas, P. (1976). Activités Physiques et Éducation Motrice. Paris: Revue EPS.

Parlebas, P. (1999). Jeux, Sports et Sociétés: Lexique de Praxéolgie Motrice. Paris: Institut du Sport et de L'éducation Physique. 
Parlebas, P. (2001). Léxico de Praxiologia Motriz juegos, deporte y sociedad. Barcelona: Editorial Paidotribo.

Potrac, P.; Jones, R., \& Armour, K. (2002). It's all About Getting Respect': the Coaching Behaviors of an Expert English Soccer Coach. Sport, Education \& Society, 2(7), 183-202. https://doi.org/10.1080/135733202200001 8869

Puig, D. S. (2011). Praxis de las acciones a balón parado en fútbol. Revisión conceptual bajo las teorias de la praxiologia motriz. 408 f. Tese (Doutorado) - Departament de Medicina i Cirurgia, Universitat Rovira i Virgili, Tarragona, Espanha.

Pujals, C. y Fiorese, L. (2002). Análise dos fatores psicológicos que interferem no comportamento dos atletas de futebol de campo. Revista da Educação Fisica/UEM, 13(1), 89-97. Recuperado de http://www.periodicos.uem.br/ojs/ index.php/RevEducFis/article/view/3756/2567

Ribas, J. F. M. (Org.). (2014). Praxiologia Motriz evoleibol: elementos para o trabalho pedagógico. Ijuí: Editora da Unijuí.

Ribas, J. F. M. (2005). Praxiologia Motriz: Construção de um Novo Olhar dos Jogos e Esportes na Escola. Motriz, 11(2), 113-120. Recuperado de https://www.rc.unesp.br/ib/efisica/motriz/11n2/10MRJ.pdf

Rodríguez Ribas, J. P. (2015). Deportes de equipo transgresores. Acción Motriz, 15, 51-62. Recuperado de https://di alnet.unirioja.es/servlet/articulo?codigo $=6324699$

Saad, M. A. (2002). Estruturação das sessões de treinamento técnico-tático nos escalões de formação do futsal. Dissertação (Mestrado em Educação Física) - Centro de Educação Física e Desporto, Universidade Federal de Santa Catarina. Florianópolis.

Sadi, R. S.; Costa, J., y Sacco, B. (2008). Ensino de esportes por meio de jogos: desenvolvimento e aplicações. Pensar a Prática, 11(1), 17-26. https://doi.org/10.5216/rpp.v11i1.1298

Sampedro Molinuevo, J. (1996). Análisis Praxiológico de los Deportes de Equipo: uma Aplicación al Fútbolsala. $261 \mathrm{f}$. Tese (Doutorado em Educação Física) - Faculdad de Ciencias de la Actividad Física y del Deporte, Universidad Politécnica de Madrid, Madrid.

Santos, E. (1979). Caderno Técnico-Didático - Futebol. Brasília: Ministério da Educação e Cultura.

Saraví, J. R. (2012). La Praxología Motriz: presente, pasado y futuro. Entrevista a Pierre Parlebas. Movimento, 18(1), 11-35. https://doi.org/10.22456/1982-8918.27065

Saraví, J. R. (2016). Prácticas Corporales Urbanas. ¿Deportes Institucionalizados O Prácticas Lúdicas? Acción Motriz, 17, 63-70. Recuperado de http://www.accionmotriz.com/documentos/revistas/articulos/17_5.pdf

Scaglia, A. J. (2015). A Pedagogia do Esporte em José Mourinho. Revista Educação e Linguagens, 4(7), 16-37. Recuperado de http://www.fecilcam.br/revista/index.php/educacaoelinguagens/article/viewFile/839/489

Scaglia, A. (2011). O futebole as brincadeiras de bola: a família dos jogos de bola com os pés. São Paulo: Phorte.

Scaglia, A.; Reverdito, R.; Leonardo, L., y Lizana, C. (2013). O ensino dos jogos esportivos coletivos: as competências essenciais e a lógica do jogo em meio ao processo organizacional sistêmico. Movimento, 19(4), 1-30. https://do i.org/10.22456/1982-8918.37893

Souza, C. B. C.; Müller, E. S.; Costa, I. T., y Graça, A. B. (2014) Quais comportamentos táticos de jogadores de futebol da categoría sub-14 podem melhorar após 20 sessões de treino? Revista Brasileira de Ciências do Esporte, 36(1), 71-86. http://doi.org/10.1590/S0101-32892014000100006

Torres, C. A. (2013). La Praxiología en El Fútbol. Incidencias Reglamentarias, Acciones Motrices, el Remate y el Gol. EFDeportes.com, Revista Digital, 18(179). Recuperado de https://www.efdeportes.com/efd179/la-praxiologiaen-el-futbol.htm

Vargas, C.E.; Saretti, D., y Bojikian, J. C. (2011). Copa do Mundo 2010 de Futebol: Análise quantitativa de gols e indicadores técnicos. Revista Brasileira de Ciências do Futebol, 5 (1), $42-46$. 